\title{
Riemann Hypothesis
}

\section{Asset Askhatbekovish Durmagambetov}

Department of Mathematics, L.N.Gumilyov Eurasian National University, Astana, Kazakhstan

Email: aset.durmagambet@gmail.com

How to cite this paper: Durmagambetov, A.A. (2017) Riemann Hypothesis. Journal of Applied Mathematics and Physics, 5, 1424-1430.

https://doi.org/10.4236/jamp.2017.57117

Received: April 22, 2017

Accepted: July 16, 2017

Published: July 18, 2017

Copyright (C) 2017 by author and Scientific Research Publishing Inc. This work is licensed under the Creative Commons Attribution International License (CC BY 4.0).

http://creativecommons.org/licenses/by/4.0/

\begin{abstract}
This work represents the development and detailing of works in [1] [2], and work is dedicated to the promotion of the results Abels obtained modifying zeta functions. The properties of zeta functions are studied; these properties lead to new regularities of zeta functions. The choice of a special type of modified zeta functions allows estimating the Riemann's zeta function and solving Riemann Problem.
\end{abstract}

\section{Keywords}

Chebyshev, Dirichlet, Riemann, Hypothesis, Zeta Function

\section{Introduction}

In this work we are studying the properties of modified zeta functions. Riemann's zeta function is defined by the Dirichlet's distribution

$$
\varsigma(s)=\sum_{n=1}^{\infty} \frac{1}{n^{s}}, s=\sigma+i t
$$

absolutely and uniformly converging in any finite region of the complex s-plane, for which $\sigma \geq 1+\epsilon, \epsilon>0$. If $\sigma>1$ the function is represented by the following Euler product formula

$$
\varsigma(s)=\prod_{p}\left[1-\frac{1}{p^{s}}\right]^{-1}
$$

where $\mathrm{p}$ is all prime numbers. $\varsigma(s)$ was firstly introduced by Euler 1737 in [3], who decomposed it to the Euler product formula (2). Dirichlet and Chebyshev, studying the law of prime numbers distribution, had considered this in [4] However, the most profound properties of the function $\varsigma(z)$ had only been discovered later, when the function had been considered as a function of a complex variable. In 1876, Riemann was the first who in [5] that:

$\varsigma(s)$ allows analytical continuation on the whole z-plane in the following form 


$$
\pi^{-s / 2} \Gamma(s / 2) s(s)=1 /(s(s-1))+\int_{1}^{+\infty}\left(x^{s / 2}-1+x^{(1-s) / 2}-1\right) \theta(x) \mathrm{d} x
$$

where $\Gamma(z)$-gamma function,

$$
\theta(x)=\sum_{n=1}^{\infty} \exp \left(-\pi n^{2} x\right)
$$

$\varsigma(s)$ is a regular function for all values of $s$, except $s=1$, where it has a simple pole with a deduction equal to 1 , and satisfies the following functional equation

$$
\pi^{-s / 2} \Gamma(s / 2) \varsigma(s)=\pi^{-(1-s) / 2} \Gamma((1-s) / 2) \varsigma(1-s)
$$

This equation is called the Riemann's functional equation.

The Riemann's zeta function is the most important subject of study and has a plenty of interesting generalizations. The role of zeta functions in the Number Theory is very significant, and is connected to various fundamental functions in the Number Theory as Mobius function, Liouville function, the function of quantity of number divisors, the fucntion of quantity of prime number divisors. The detailed theory of zeta functions is showed in [6]. The zeta function spreads to various disciplines and now the function is mostly applied in quantum statistical mechanics and quantum theory of pole [7] [8] [9]. Riemann's zeta function is often introduced in the formulas of quantum statistics. A well-known example is the Stefan-Boltzman law of a black body's radiation. The given aspects of the zeta function reveal global necessity of its further investigation.

We are interested in the analytical properties of the following generalizations of zeta functions:

$$
\begin{gathered}
P(s)=\sum_{j \geq 1} \frac{1}{p_{j}^{s}}, P_{m}(s)=\sum_{j=1}^{m} \frac{1}{p_{j}^{s}}, P^{m}(s)=\sum_{j>m} \frac{1}{p_{j}^{s}}, \\
\varsigma_{p}^{m}(z)=\varsigma(s)-P^{m}(s), \varsigma^{m}(s)=\sum_{n=1}^{m} \frac{1}{n^{s}}, s=\sigma+i t .
\end{gathered}
$$

where $p_{j}$ runs through all prime numbers in ascending order. The forms of the given function $P(s)$ allow assuming that they possess the same properties as the zeta function, but it is not quite obvious, considering

$$
\ln (\varsigma(s))=\sum_{n=1}^{\infty} P(n s) / n, f(s)=\ln (\varsigma(s))-P(s)
$$

as $\operatorname{Re}(s)>1 / 2+\epsilon$ we have

$$
|f(s)|=\left|\sum_{m=2}^{\infty} P(m s) / m\right| \leq \sum_{m=2}^{\infty}|P(m s) / m| \leq C_{\varepsilon} \sum_{m=2}^{\infty}\left|2^{-m \epsilon} / m\right|<C C_{\epsilon}<\infty
$$

we see the necessity of analyzing $P(s)$ for a deeper understanding of the properties of zeta functions.

\section{Results}

These are the well-known Abel's results.

Theorem 1. Let the function $\phi(x)$ be limited on every finite interval, and 


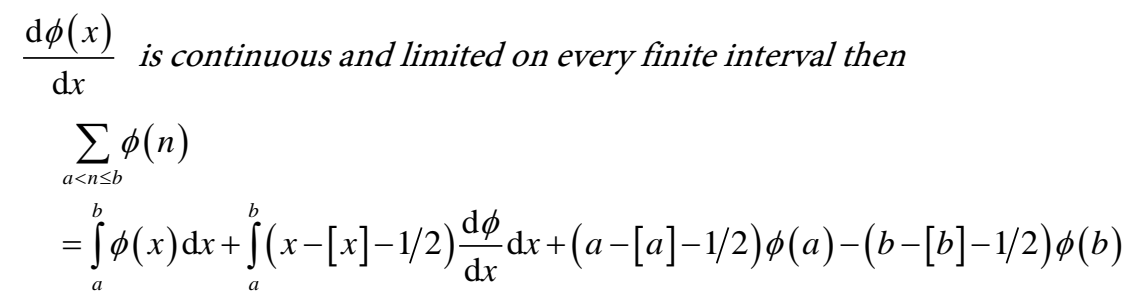

Let $N$ be the set of all natural numbers.

Corollary 1. Let the function $\phi(x)=x^{-s}$, Res $>0, a, b \in N$ then

$$
\sum_{a<n \leq b} n^{-s}=\frac{b^{1-s}-a^{1-s}}{1-s}-s \int_{a}^{b} \frac{(x-[x]-1 / 2)}{x^{s+1}} \mathrm{~d} x+\frac{1}{2}\left(b^{-s}-a^{-s}\right)
$$

Corollary 2. Let the function $\phi(x)=x^{-s}$, Res $>0, a=1, b=\infty$ then

$$
\sum_{1<n<\infty} n^{-s}=-\frac{1}{1-s}-s \int_{1}^{\infty} \frac{(x-[x]-1 / 2)}{x^{s+1}} \mathrm{~d} x-\frac{1}{2}
$$

Corollary 3. Let the function $\phi(x)=x^{-s}$, Res $>0, p_{j}, p_{j+1} \in N$ then

$$
\begin{aligned}
\sum_{p_{j}<n \leq p_{j+1}-1} n^{-s}= & \frac{\left(p_{j+1}-1\right)^{1-s}-p_{j}^{1-s}}{1-s}-s \int_{p_{j}}^{p_{j+1}-1} \frac{(x-[x]-1 / 2)}{x^{s+1}} \mathrm{~d} x \\
& +\frac{1}{2}\left(\left(p_{j+1}-1\right)^{-s}-p_{j}^{-s}\right)
\end{aligned}
$$

We formulated Abel's results for the variable s from the complex plane with the only condition Res $>0$ We note once again that such formulations imply an analytic continuation of the zeta- function to the complex plane and we will use such formulas for the analytic continuation of the modified zeta-function $P^{m}(s)$.

For the function $P^{m}(s)$, let us apply the results obtained by Abel's for the zeta function representation. For simplicity, we formulate several lemmas.

Lemma 1. Let

$$
\begin{gathered}
|s|<1 / \epsilon, \epsilon>0, \operatorname{Re}(s)>-1+\epsilon \\
P_{\Delta}(s)=\sum_{j>m}\left[\frac{1}{p_{j}^{s}}-\frac{1}{\left(p_{j}-1\right)^{s}}\right]+s P^{m}(s+1),
\end{gathered}
$$

then

$$
\left|P_{\Delta}(s)\right|<C_{\epsilon}<\infty \text {. }
$$

Proof.

$$
\begin{aligned}
& \frac{1}{p_{j}^{s}}-\frac{1}{\left(p_{j}-1\right)^{s}}+\frac{s}{p_{j}^{s+1}}=\int_{p_{j}-1}^{p_{j}} \mathrm{~d} x^{-s}+\frac{s}{p_{j}^{s+1}}=s \int_{p_{j}-1}^{p_{j}}\left[-\frac{1}{x^{s+1}}+\frac{1}{p_{j}^{s+1}}\right] \mathrm{d} x \\
& s \int_{p_{j}-1}^{p_{j}}\left[-\frac{1}{x^{s+1}}+\frac{1}{p_{j}^{s+1}}\right] \mathrm{d} x=s \int_{p_{j}-1}^{p_{j}} \int_{x}^{p_{j}} \mathrm{~d} \tau^{-s-1} \mathrm{~d} x=-s(s+1) \int_{p_{j}-1}^{p_{j}} \int_{x}^{p_{j}} \tau^{-s-2} \mathrm{~d} \tau \mathrm{d} x
\end{aligned}
$$

from the last equation we obtain: 


$$
\left|P_{\Delta}(s)\right|<\left|\sum_{j>m} s(s+1) \int_{p_{j}-1}^{p_{j}} \int_{x}^{p_{j}} \tau^{-s-2} \mathrm{~d} \tau \mathrm{d} x\right|<2 \sum_{j>m} \frac{|s|^{2}}{\left(p_{j}-1\right)^{1+\epsilon}}<C_{\epsilon}<\infty .
$$

The last lemma allows us to consider $P_{\Delta}(s)$ as an analytic function of the complex variable $s$ in the domain $\operatorname{Re}(s)>-1$.

Corollary 4. Let $|s|<1 / \epsilon, \epsilon>0, \operatorname{Re}(s)>\epsilon$ then for the function

$$
\Delta(s)=\sum_{j>m}\left[\frac{1}{\left(p_{j}\right)^{s}}-\frac{1}{\left(p_{j}-1\right)^{s}}\right],
$$

we have

$$
\Delta(s)=-s \sum_{j>m} \frac{1}{p_{j}^{s+1}}+P_{\Delta}(s) .
$$

Proof. According to the corollary conditions we have from last lemma we have proof the corollary.

The last lemma allows us to consider $\Delta(s)$ as an analytic function of the complex variable $s$ in the domain $\operatorname{Re}(s)>0$.

Lemma 2. For the function

$$
\Theta(s)=s \sum_{j>m} \int_{p_{j-1}-1}^{p_{j}}\left[\frac{x}{x^{s+1}}-\frac{[x]}{x^{s+1}}-\frac{1 / 2}{x^{s+1}}\right] \mathrm{d} x
$$

as $\operatorname{Re}(s)>1$ we have:

$$
\Theta(s)=s\left[\frac{\Delta(s-1)}{1-s}-\frac{\Delta(s-1)}{s}+\frac{P^{m}(s)}{s}-\frac{\Delta(s)}{2 s}\right]
$$

Proof. Follows from computing of integrals.

\section{Lemma 3. For the function}

$\Delta(s-1)$, as $\operatorname{Re}(s)>1$, we have:

$$
\begin{gathered}
-\Delta(s-1)-m^{1-s}=\sum_{j>m}\left(\left(p_{j+1}-1\right)^{1-s}-p_{j}^{1-s}\right) \\
\sum_{j>m} s \int_{p_{j}}^{p_{j+1}-1} \frac{(x-[x]-1 / 2)}{x^{s+1}} \mathrm{~d} x=s \int_{m}^{\infty} \frac{(x-[x]-1 / 2)}{x^{s+1}} \mathrm{~d} x-\Theta(s) ;
\end{gathered}
$$

Proof. Computing the sums, we have

$$
\begin{aligned}
\sum_{j>m}\left(p_{j+1}-1\right)^{1-s}-p_{j}^{1-s} & =-m^{1-s}+\sum_{j>m}\left(\left(p_{j}-1\right)^{1-s}-p_{j}^{1-s}\right) \\
& =-m^{1-s}-\sum_{j>m}\left(\frac{1}{p_{j}^{s-1}}-\frac{1}{\left(p_{j}-1\right)^{s-1}}\right) \\
& =-m^{1-s}-\Delta(s-1)
\end{aligned}
$$

The second assertion follows from the properties of the integral. 
Theorem 2. Let the function

$\operatorname{Re}(s)>1$, then

$$
\begin{aligned}
2 s P^{m}(s)= & \varsigma(s)-\varsigma^{m}(s)+\frac{m^{1-s}}{1-s}+s \int_{m}^{\infty} \frac{(x-[x]-1 / 2)}{x^{s+1}} \mathrm{~d} x \\
& +\frac{1}{2}\left(\Delta(s)+m^{-s}\right)+2 P_{\Delta}(s-1)
\end{aligned}
$$

Proof. Using Corollary 3 we have

$$
\begin{aligned}
\varsigma_{p}^{m}(s)= & \varsigma^{m}(s)+\sum_{j>m} \sum_{p_{j}<n \leq p_{j+1}-1} n^{-s}=\varsigma^{m}(s)+\sum_{j>m} \frac{\left(p_{j+1}-1\right)^{1-s}-p_{j}^{1-s}}{1-s} \\
& -\sum_{j>m} s \int_{p_{j}}^{p_{j+1}-1} \frac{(x-[x]-1 / 2)}{x^{s+1}} \mathrm{~d} x+\frac{1}{2} \sum_{j>m}\left(\left(p_{j+1}-1\right)^{-s}-p_{j}^{-s}\right) .
\end{aligned}
$$

Using Lemma 1-3 we have

$$
\begin{aligned}
\varsigma_{p}^{m}(s)= & \varsigma^{m}(s)-\frac{1}{1-s}\left(\Delta(s-1)+m^{1-s}\right) \\
& -s \int_{m}^{\infty} \frac{(x-[x]-1 / 2)}{x^{s+1}} \mathrm{~d} x+\Theta(s)-\frac{1}{2}\left(\Delta(s)+m^{-s}\right)
\end{aligned}
$$

Using Lemma 3 we have

$$
\begin{aligned}
\varsigma_{p}^{m}(s)= & \varsigma^{m}(s)-\frac{1}{1-s}\left(\Delta(s-1)+m^{1-s}\right)-s \int_{m}^{\infty} \frac{(x-[x]-1 / 2)}{x^{s+1}} \mathrm{~d} x \\
& +s\left[\frac{\Delta(s-1)}{1-s}-\frac{\Delta(s-1)}{s}+\frac{P^{m}(s)}{s}-\frac{\Delta(s)}{2 s}\right]-\frac{1}{2}\left(\Delta(s)+m^{-s}\right)
\end{aligned}
$$

Simplifying the last expression, we obtain

$$
\begin{aligned}
\varsigma_{p}^{m}(s)= & \varsigma^{m}(s)+\Delta(s-1)\left[\frac{s-1}{1-s}-1\right]+P^{m}(s)-\frac{m^{1-s}}{1-s} \\
& -s \int_{m}^{\infty} \frac{(x-[x]-1 / 2)}{x^{s+1}} \mathrm{~d} x-\frac{1}{2}\left(\Delta(s)+m^{-s}\right)
\end{aligned}
$$

Simplifying the last expression, we obtain

$\varsigma(s)=\varsigma^{m}(s)-2 \Delta(s-1)+2 P^{m}(s)-\frac{m^{1-s}}{1-s}-s \int_{m}^{\infty} \frac{(x-[x]-1 / 2)}{x^{s+1}} \mathrm{~d} x-\frac{1}{2}\left(\Delta(s)+m^{-s}\right)$

Using Lemma 1 we have

$$
\begin{aligned}
\varsigma(s)= & s^{m}(s)-2 P_{\Delta}(s-1)+2(s-1) P(s)+2 P^{m}(s) \\
& -\frac{m^{1-s}}{1-s}-s \int_{m}^{\infty} \frac{(x-[x]-1 / 2)}{x^{s+1}} \mathrm{~d} x-\frac{1}{2}\left(\Delta(s)+m^{-s}\right)
\end{aligned}
$$

Using analytic properties functions $\varsigma(s), \varsigma^{m}(s), P_{\Delta}(s)$ from the last equation we obtain $P(s)$ as an analytic function of the complex variable $s$ in the domain $\operatorname{Re}(s)>0$.

Computing $s P^{m}(s)$, we obtain 


$$
\begin{aligned}
& 2 s P^{m}(s) \\
& =\varsigma(s)-\varsigma^{m}(s)-\left[-\frac{m^{1-s}}{1-s}-s \int_{m}^{\infty} \frac{(x-[x]-1 / 2)}{x^{s+1}} \mathrm{~d} x-\frac{1}{2}\left(\Delta(s)+m^{-s}\right)-2 P_{\Delta}(s-1)\right]
\end{aligned}
$$

From the last equation we obtain the regularity of the function $P^{m}(s)$ as $0<\operatorname{Re}(s)<1$.

Theorem 3. The Riemann's function has nontrivial zeros only on the line $\operatorname{Re}(s)=1 / 2$;

Proof. For $f(s)=\sum_{m=2}^{\infty} P(m s) / m$, as $\operatorname{Re}(s)>1 / 2+\epsilon$, we have

$$
|f(s)|=\left|\sum_{m=2}^{\infty} P(m s) / m\right| \leq \sum_{m=2}^{\infty}|P(m s) / m| \leq C_{\epsilon} \sum_{m=2}^{\infty}\left|2^{-m \epsilon} / m\right|<C C_{\epsilon}<\infty
$$

Applying the formula from the theorem 2

$$
\ln (s(s))=P(s)+\sum_{m=2}^{\infty} P(m s) / m=P(s)+f(s)=P_{m}(s)+P^{m}(s)+f(s)
$$

estimating by the module

$$
|\ln (\varsigma(s))| \leq\left|P^{m}(s)\right|+|f(s)|+\left|P_{m}(s)\right| .
$$

Estimating the zeta function, potentiating, we obtain

$$
|\varsigma(s)|>\exp \left[-\left|P^{m}(s)\right|-|f(s)|-\left|P_{m}(s)\right|\right]
$$

According to Theorem 2, $\left|P^{m}(s)\right|$ limited for s from the following set

$$
(s,|s|<1 / \epsilon,|s-1|>\epsilon, \epsilon>0)
$$

finally we obtain:

$$
|\varsigma(s)|>\exp \left[-C_{\epsilon}\right], \operatorname{Re}(s)>1 / 2+1 / \epsilon,|s|<1 / \epsilon,|s-1|>\epsilon, \epsilon>0
$$

These estimations for $|P(s)|,|f(s)|,\left|P_{m}(s)\right|$ prove that function does not have zeros on the half-plane $\operatorname{Re}(s)>1 / 2+\epsilon$ due to the integral representation (3) these results are projected on the half-plane $\operatorname{Re}(s)<1 / 2$. Due to the arbitrariness of the $\epsilon$, we obtain a proof of the Riemann hypothesis. Riemann's hypothesis is proved.

\section{Conclusion}

In this work we obtained the estimation of the Riemann's zeta function logarithm outside of the line $\operatorname{Re}(s)=1 / 2$ and outside of the pole $s=1$. This work accomplishes all the works of the greatest mathematicians, applying their immense achievements in this field. Without their effort we could not even attempt to solve the problem.

\section{Acknowledgements}

The author thanks the National Engineering Academy of the Republic of Kazakhstan in the person of Academician NAS RK Zhumagulov for constant attention and support. 
The author thanks prof. S.N Baibekov for introducing the prime numbers to the problematics in the collective article [10]. Without this the work would be impossible.

The author thanks the seminar on Mathematics Kazakhstan branch of the Moscow State University of attention and valuable comments.

The author thanks Professor M.O Otelbaev for detailed review and valuable comments.

\section{References}

[1] Durmagambetov, A.A. (2016) The Riemann Hypothesis-Millennium Prize Problem. Advances in Pure Mathematics, 6, 915-920. https://doi.org/10.4236/apm.2016.612069

[2] Durmagambetov, A.A. (2017) The Solution of the Riemann Hypothesis. AIP Conference Proceedings, 1836, 020030. https://doi.org/10.1063/1.4981970

[3] Euler, L. (1988) Introduction to Analysis of the Infinite by John Blanton. Book I, ISBN 0-387-96824-5, Springer-Verlag, Berlin.

[4] Chebyshev P.L. (1946) Fav. Mathematical Works M.-L..

[5] Riemann, G.F.B. (1972) On the Number of Prime Numbers Less than a Given Quantity. Chelsea, New York.

[6] Titchmarsh, E.C. (1986) The Theory of the Riemann Zeta Function. Second Revised (Heath-Brown) Edition. Oxford University Press, Oxford.

[7] Ray, D., Singer, I.M. (1971) R-Torsion and the Laplacian on Riemannian Manifolds. Advances in Mathematics, 7, 145-210. https://doi.org/10.1016/0001-8708(71)90045-4

[8] Bost J.-B. Fibres determinants, determinants regularises et measures sur les espaces de modules des courbes complexes. Sem. Bourbaki, 39 eme annee 1986-1987.

[9] Kawagoe, K., Wakayama, M. and Yamasaki, Y. (2008) The q-Analogues of the Riemann Zeta, Dirichlet L-Functions, and a Crystal Zeta-Function. Forum Math, Vol. 77.

[10] Baibekov, S.N. and Durmagambetov, A.A. (2016) Infinitely Many Twin. arXiv, 71, 1609.04646.

\section{Submit or recommend next manuscript to SCIRP and we will provide best} service for you:

Accepting pre-submission inquiries through Email, Facebook, LinkedIn, Twitter, etc. A wide selection of journals (inclusive of 9 subjects, more than 200 journals)

Providing 24-hour high-quality service

User-friendly online submission system

Fair and swift peer-review system

Efficient typesetting and proofreading procedure

Display of the result of downloads and visits, as well as the number of cited articles

Maximum dissemination of your research work

Submit your manuscript at: http://papersubmission.scirp.org/

Or contact jamp@scirp.org 